\title{
Gauteng's disaster management clinicians outline World Cup shortfalls
}

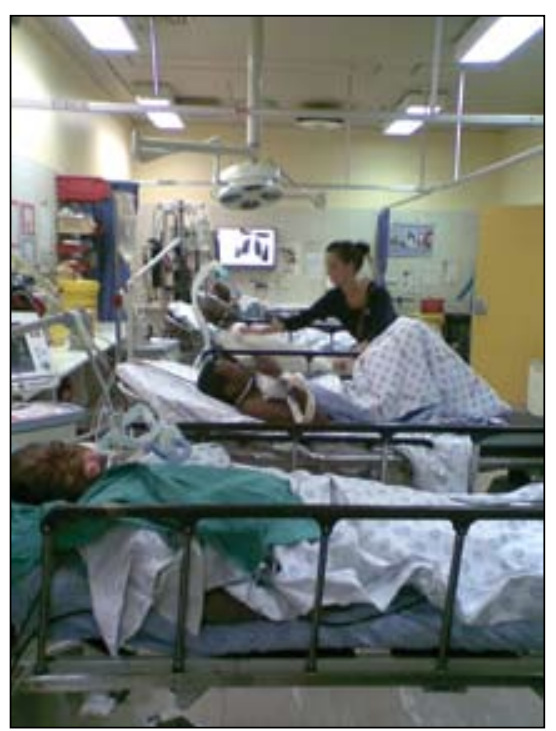

A lack of standardised disaster management protocols, poor intercommunication between health care authorities and emergency rescue services and shaky environmental health and port health controls threaten Gauteng's risk preparedness for the $\mathbf{2 0 1 0}$ World Cup.

This emerged from interviews with the top clinicians responsible for disaster management at Charlotte Maxeke Johannesburg Academic, Chris Hani Baragwanath and Helen Joseph hospitals last month.

While making herculean efforts to ensure they are as prepared as possible by June, the hands-on managers admitted that it would take 'some stepping up' to handle a 'surge' beyond their current near-peak capacity operations on any difficult weekend.

Depending on which public hospital Izindaba spoke to, the challenges came down to shortages of clinical and support staff, equipment, and ICU and trauma nursing skills, and budget shortfalls.

Off-site 1 - 3-day basic disaster management training courses and in-house emergency care training have helped prepare the less specialised health care work force, thanks to private health care sector generosity and public sector clinical manager innovation, respectively. Fortunately the veteran clinicians can lean on a relatively large body of well-trained junior doctors, thanks to the inclusion in most medical faculty curricula of trauma and emergency medicine and the relatively recent registration of emergency medicine as a specialty. (The first handful of emergency medicine specialists qualified in 2007.)

These innovations have now paid handsome dividends, putting local training in this field well above the world average, according to a World Health Organization (WHO) survey.

However, on the equipment front, the 'Cinderella' of the Gauteng hospital trio, the ageing and under-funded 557-bed Helen Joseph (designed as a secondary hospital but on the academic training circuit), had no manual blood pressure machine, one functional ventilator and a cannabilised defibrillator in its emergency unit at the time of writing. Basic patient essentials such as sheets and blankets were in short supply, while electronic monitoring equipment was described as 'partially functional'.

While making herculean efforts to ensure they are as prepared as possible by June, the hands-on managers admitted that it would take 'some stepping up' to handle a 'surge' beyond their current near-peak capacity operations on any difficult weekend.

'We manage, but it's not correct patient care. If we don't have the equipment in place and additional nursing staff appropriately trained by June we cannot be accredited but I won't let that happen,' said a determined Dr Adrienne Wulfsohn.

Wulfsohn was seconded from Gauteng Province's Health Central Office in December to upgrade the hospital's disaster planning, and faces an uphill battle.
Doctors at Helen Joseph have become role models for keeping detailed notes 'to protect our backs', should any adverse events occur. The hospital's clinical director, Dr Geraldine Kirby, said the dire shortage of beds, particularly ICU beds, lack of support and clinical staff and equipment, and the poor state of the building were major impediments.

'If we're hardly coping day to day how are we going to cope if World Cup surge capacity is required?,' she asked before referring specific enquiries to Wulfsohn.

\section{Ambulance diversions; comms critical}

Wulfsohn put Helen Joseph Hospital on 'divert' (diverting incoming ambulances because of lack of overall hospital capacity) three times between December and early this February.

She explains: 'First, road construction guys working at the hospital entrance burst the water main (the city sent water tankers and they were re-opened within an hour and a half). Then we went on divert for 40 minutes last week (beginning of February) with 7 resuscitations and 8 incoming taxi patients - I just didn't have the physical beds to put the patients on - but they brought them anyway because we were nearest. Because of poor communication, suddenly with no forewarning, you're sitting with 7 resuscitations,' she explained.

Wulfsohn said she and her staff relied on personal cell phones to communicate with pre-hospital services and on-call colleagues. There were several 'dead (reception) spots' in the hospital, including the resuscitation room (which could handle 8 patients at a time).

For optimal communication with pre-hospital services she would need 4 radios -1 each for provincial authorities, local authorities, the private sector and the rescue helicopter. 'The private sector seems to be able to standardise more easily but it's harder in the public sector.' 
Each local authority also had its own call centre linked to various ambulance services but did not 'talk to' the provincial call centre, while none of the call centres were directly linked to the hospitals (i.e. no radios at hospitals). This had serious implications when it came to co-ordinating ambulance diversions between World Cupaccredited hospitals and the time-critical triage of patients.

A state-of-the-art local government disaster management call centre is situated in the same building as the provincial call centre, yet the two are not directly linked.

\section{'Double triage' caused alarming delays}

Wulfsohn, a veteran of 26 years in emergency medicine, is adapting her hospital triage system to a prehospital one because 'sometimes I've found 5 or 6 ambos parked outside the hospital with patients, waiting for us to re-triage'.

Professor Jacques Goosen, head of trauma at the 1088 -bed Charlotte Maxeke Johannesburg Academic Hospital, said that the 'vicarious violence around taxis, beer and fan parks' far exceeded anything the stadiums could throw at their units. Yet emergency medicine systems seemed to have been designed around the stadia 'while the huge party that goes on elsewhere ends up in the public hospitals'.

Goosen described the Confederations Cup final last year (a Sunday) as having provided his hospital with 'the perfect storm' because of their lack of surge capacity at the time. 'We had 9 patients ventilated in casualty and our ICUs were full,' he revealed.

However, a subsequent internal 'post mortem', which also drew on 3 other major trauma-inducing events over the past 9 years, had resulted in a disaster management plan with regard to which clinicians were finally breathing a cautious sigh of relief.

'Our new MEC (Quedani Mahlangu) went begging for money for equipment that has enabled us to double our ventilated beds within a few hours. We have 8 ventilated beds and can now double and treble that within $6-8$ hours (i.e. stabilised and moved on to ICU or high care),' he explained.

\section{Doctor staffing better, nurse availability a problem}

An additional boost to their capacity was the number of community service doctors who had chosen to stay on and fill posts which were $50 \%$ vacant (hospital wide) at the time of the Confederations Cup.

'We're also starting to fill nursing posts - what came out of the post mortem was that you can't call in nurses because they stay far away and communications are difficult.'

The solution was to call in $20-30$ extra nurses in advance on 'event' days and 'bad' weekends and pay them overtime to strengthen shifts. The resulting cost-benefit ratio meant that incoming patients could spend up to 10 days less in an ICU (1 ICU day costs the province R15 000 - the approximate cost of 10 nursing shifts). The better qualified nurses would be 'drawn down' from the wards into the ICUs while others helped 'decant' existing non-serious patients to nearby drainage hospitals and handled new arrivals.

Goosen said that without the private and NGO sectors the region's hospitals would be disastrously underprepared.

He confirmed that over the last New Year's weekend Charlotte Maxeke Johannesburg Academic Hospital's emergency unit dealt with 52 'priority 1 ' (immediate life-threatening injury) patients - far more than most reported mass casualty events. 'We called a "code yellow" (calling in off-duty trauma doctors and nurses) but the matron vetoed it because no nurse would arrive in time; so we learnt some valuable lessons.'

Goosen said that because Gauteng's budget 'ran out' towards the end of last year, Netcare had stepped in to put as many public sector GPs as possible from all provincial hospitals through a licensed Major Incident Medical Management course.
The Department of Medicine at Wits University had also put 150 doctors through its emergency medicine course, training them in managing mass casualties.

\section{Private and NGO sector to the rescue}

'Apart from that there are a lot of major in-house training initiatives at the major public hospitals, so with limited support from government we've got a lot of people trained,' he added. Goosen said that without the private and NGO sectors the region's hospitals would be disastrously under-prepared.

Charlotte Maxeke Johannesburg Academic is also increasing the number of available theatre nurses so that the hospital can run up to 5 theatres instead of the current 3. Credit was due to a former clinician at the hospital, West Indian Dr Ricardo Hamilton, who had completed his MMed thesis on improving the hospital's disaster management systems. Hamilton analysed the factors causing closure of the hospital's trauma unit in 2007, the hospital's experience of the April 2001 Ellis Park Soccer Stadium stampede (146 patients treated in just over 2 hours with no mortalities), the East Rand xenophobic violence of 17 - 18 May 2008 (24 patients ventilated, 56 priority 1 and 150 priority $2-3$ handled in 48 hours), the patient influx during the Confederations Cup final, and last New Year's eve. 'That all showed exactly how badly prepared we were ... that we could run a trauma service but not a system, while the Confed Cup showed us that we lacked surge capacity. Hamilton told us why this was and what to do about it.

Dr Frank Plani, deputy trauma chief and principal surgeon at the 2800 -bed Chris Hani Baragwanath Hospital, was putting the finishing touches to his disaster plan.

With a brand-new, well-equipped trauma unit where the official 8-bed capacity has already been pushed to 15 , a resuscitation room capable of ventilating up to 32 patients, and another 6-bed medical resuscitation room, he has a platform to build on.

The most important finding of his disaster and resuscitation research (part 
of a thesis he's writing), is that 'we'd not be able to respond in the hospital disaster phase without a large number of nurses' - and that mobilising them is a problem.

Plani cited last New Year's eve when they mobilised all their available doctors to deal with 35 resuscitation patients at once while another 50 patients waited outside.

'We had just 4 trauma-trained nurses whose mobilisation was a problem, but we supplemented them with less suitably trained ones, I can't remember the exact numbers,' he confessed.

Chris Hani Baragwanath Hospital would this June 'double up on everybody, nurses in theatre, basically everybody works double shift on the few days that the World Cup affects us directly'. They were head hunting senior nurses who worked at Bara during the 1984/1985 riots to help prioritise the most needed equipment, but corporate sponsorship would be essential to secure it.

His plan includes basic disaster packs attached to 8 dedicated specialised trolleys, each with a doctor and volunteer, working in rotation with dedicated junior nurses doing theatre hand-overs for continuity of care. 'We're busy with a skills audit and have taken all our surgeons through ultrasound and EM courses,' he added.

Plani said the main difference between Chris Hani Baragwanath and Charlotte Maxeke Johannesburg Academic was that the latter had 'finite space', while his hospital had 'almost unlimited space but fewer resources'. 'So for hundreds of patients we'll adopt a "treat as you find" (pre-hospital) approach with triage in a big parking lot next to the entrance while our huge outpatient department takes all the walking wounded.' He presented his disaster plan to the hospital executive and their CEO in early February and they 'seemed very keen on it'.

\section{What about medical emergencies?}

The hospital disaster chiefs were less confident about what would happen should a large-scale medical emergency coincide with the expected trauma surge, and some pointed to port control and environmental health weaknesses in Government.

Wulfsohn said VIPs were ironically among the biggest port control health safety threats as they were given special dispensation on yellow fever vaccination certificates that enabled them to receive a vaccination only upon entering the country.

This was in spite of the required 10-day - 2-week post-immunisation waiting period.

'Will soccer teams also be considered VIPs?,' she asked pointedly.

Dr Patrick Moonasar, director of malaria and other vector-borne diseases, responded that all international health requirements would be 'strictly complied with'.

\section{'However, we live in the} real world and if we have a head of state or other such VIP from any endemic area (arriving unvaccinated), a top-level political decision will have to be made based on their value to us and then strict surveillance instituted.'

Any VIP exceptions to this held the potential to 'open up a can of worms for everybody else'.

'However, we live in the real world and if we have a head of state or other such VIP from any endemic area (arriving unvaccinated), a top-level political decision will have to be made based on their value to us and then strict surveillance instituted.'

Identical surveillance would be imposed on any passenger for whom a required vaccination was contraindicated. 'But these would be absolute exceptions to the rule,' he emphasised.

Dr Peter Fuhri, national disaster management co-ordinator, said all air and maritime carriers were being asked to tighten up passenger health questionnaires and to notify South African authorities well in advance of any 'unwell' passengers. Environmental health staff training and numbers would be beefed up at all ports of

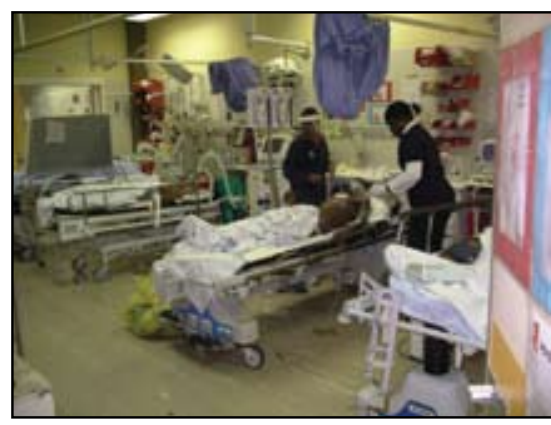

Patients being prepared for transfer to the ICU at Johannesburg General Hospital.

entry and supplemented by Centre for Disease Control staff. The WHO would deploy a disease surveillance expert to the national health department for the duration of the World Cup.

Disease outbreak response protocols had been reviewed and outbreak teams had received extra training while pre-event inspections of all fan parks and public viewing areas were being undertaken by the responsible municipal health services.

He said the chief lessons learnt from the Confederations Cup included a need for tightening up command and control structures and ensuring better and more specific stadia access accreditation for relevant medical and health support staff.

Patient delivery to relevant hospitals (there is a grading system with FIFA officials and other VIPs, those with travel insurance and medical aids assigned to various private hospitals and others to public hospitals) would be determined by the manager in the relevant provincial emergency medical services communications centre.

Responding to the surge capacity concerns of his Gauteng public hospital colleagues, Fuhri replied: 'That'll always be a difficult one. Nobody in their right mind thinks public hospitals can be transformed overnight or even in 3 years. 2010 is not the answer. We just have to be intelligent in terms of strategies - and they're doing that.'

Professor Goosen summed up preparedness sentiment thus: 'As South Africans we always seem to do stuff at the very last minute, but I think we're getting our emergency plans right at last.'

Chris Bateman 